\title{
Third-Person-Effekte bei der Wahrnehmung von Schönheitsidealen durch Frauen und Männer
}

Marco Dohle

Die mediale Darstellung von Frauen und Männern mit idealisiertem Ausseben kann Wirkungen ausüben, etwa auf die Zufriedenheit von Rezipientinnen und Rezipienten mit ibrem Ausseben. Laut Theorien indirekter Medienwirkungen wie dem Third-PersonEffekt ist dafür nicht nur die eigene Sicht auf die idealisierten Medienbilder zentral, sondern die Erwartung, dass andere Personen die Frauen und Männer in den Medien attraktiv finden. Daraus entwickelt sich die Sorge, von anderen mit diesen Akteuren verglichen zu werden - mit negativem Ergebnis für die eigene Person. Dies kann beispielsweise den Wunsch auslösen, das eigene Äußere zu verändern. Um dies empirisch zu testen, wurden weiblichen und männlichen Probanden Bilder weiblicher oder männlicher Models vorgelegt. Eingeschätzt werden sollten Einflüsse dieser Bilder auf die eigene Person sowie auf andere Franen und Männer. Als Ergebnis zeigt sich unter anderem, dass anderen Personen größere Wirkungen zugeschrieben werden als sich selbst. Insbesondere Frauen wird eine starke Beeinflussung unterstellt, vor allem als Folge der Rezeption von Bildern mit weiblichen Models. Ferner wurde überprüft, ob die Wabrnebmungen Konsequenzen haben - zum Beispiel auf den Wunsch, das eigene Ausseben durch das Einhalten von Diäten zu verändern. Dies war jedoch nur sehr partiell der Fall.

Schlagwörter: Medienwirkungen, Third-Person-Effekt, wahrgenommene Medieneinflüsse, Schönheitsideale, Experiment

\section{Einleitung}

Das Aussehen von Frauen und Männern in Filmen, Fernsehserien oder in der Werbung entspricht selten dem der Normalbevölkerung, sondern eher bestimmten Schönheitsidealen. Den Ergebnissen von Inhaltsanalysen zufolge sind Frauen zum Beispiel in fiktionalen Fernsehserien deutlich häufiger schlank als in der Realität (Greenberg et al. 2003). Medial dargestellte Männer sind dagegen oft sehr muskulös - und dies im Vergleich zu früheren Zeitpunkten in zunehmendem Maße (Law \& Labre 2002).

Solche medial vermittelten Schönheitsideale, vor allem die Schlankheitsideale, sind Gegenstand kritischer Diskussionen (im Überblick: Baumann 2009, Harrison 2009). Hauptgrund dafür ist die Befürchtung, die Darstellung attraktiver Frauen und Männer führe bei Mediennutzern zu einer Unzufriedenheit mit dem eigenen Aussehen. Bisweilen wird die Darstellung von schlanken Frauen sogar für die Entstehung von Essstörungen bei Rezipientinnen mitverantwortlich gemacht (Harrison 2001, Bissell \& Zhou 2004). Ergebnisse einer Metaanalyse bisheriger Studien verdeutlichen, dass mediale Einflüsse auf die Körperzufriedenheit und auf das Essverhalten von Frauen, obgleich auf eher moderatem Niveau, auch durchaus zu finden sind (Grabe et al. 2008).

Es ist daher relevant, Wirkungen der Darstellungen etwa von Models in Zeitschriften oder in der Werbung zu untersuchen. Etwaige Wirkungen basieren auf komplexen Prozessen, die neben den Medieninhalten unter anderem situative Faktoren, die Persönlichkeit von Mediennutzern und soziale Kontexte einschließen. Entsprechend zentral ist die Frage, warum bei Rezipientinnen und Rezipienten zum Beispiel eine Unzufriedenheit mit dem eigenen Aussehen entsteht. Ein möglicher Erklärungsansatz ist dabei der Third-Person-Effekt (Davison 1983): Mediennutzer - so der Kerngedanke - sind der 
Ansicht, dass andere Personen (stärker als sie selbst) von den Schönheitsdarstellungen in den Medien beeinflusst werden und sie selbst im Vergleich mit den Medienakteuren bewerten. Diese von anderen durchgeführten Vergleiche, so die Befürchtung, fallen zuungunsten der eigenen Person aus. Dies kann in der Folge zur Unzufriedenheit mit dem Aussehen führen.

In einer experimentell angelegten Studie wurde dies überprüft. Die Befragten sollten angeben, welche Wirkungen der Darstellung idealisierter Medienakteure sie auf sich selbst und auf andere Personengruppen einschätzen. Ferner wurden mögliche Folgen dieser Einschätzungen auf der Individualebene gemessen. Solche Studien liegen bereits vor (z. B. David et al. 2002, Chia 2007). Befragte waren dabei Frauen. An der vorliegenden Untersuchung nahmen jedoch Frauen und Männer teil. Ihnen wurden zudem entweder Bilder weiblicher oder männlicher Fotomodelle vorgelegt. Eingeschätzt werden sollten Einflüsse dieser Bilder auf die eigene Person, aber auch auf andere Frauen und auf andere Männer. Auf diese Weise konnten Einflusswahrnehmungen verglichen und auf ihre möglichen Konsequenzen hin überprüft werden. So ließ sich etwa kontrollieren, ob gemäß der Third-Person-Forschung (Meirick 2005) stets Personen des anderen Geschlechts die stärkste Beeinflussung unterstellt wird, ob immer Frauen für beeinflussbarer gehalten werden (Jones 2001) oder ob dies vom Geschlecht der präsentierten Models abhängt.

\section{Theoretische Grundlagen}

\subsection{Medial vermittelte Schönheitsideale: Wirkungsannabmen}

Zu der Frage, warum medial vermittelte Schönheitsideale Auswirkungen auf Rezipientinnen und Rezipienten haben können, liegen verschiedene Erklärungsansätze vor. Sie gelten primär für negative Wirkungen. Dazu zählt eine Unzufriedenheit mit dem eigenen Aussehen, die wiederum zum Wunsch führen kann, dieses zu verändern. Sehr starke Wirkungen würden vorliegen, wenn die Medienrezeption als ein Grund für die Entstehung von Essstörungen gelten muss (es können jedoch durchaus auch positive Wirkungen auftreten, zum Beispiel eine Verbesserung der eigenen Stimmung; siehe dazu: Baumann 2009).

Eine Grundlage für Wirkungsvermutungen sind zunächst die Medieninhalte selbst. In den Medien abgebildete oder auftretende Personen entsprechen Inhaltsanalysen zufolge idealisierten Körperbildern (z. B. Law \& Labre 2002, Spitzer et al. 1999): Frauen sind, so das aktuelle Ideal, vor allem schlank, Männer durchtrainiert und muskulös (Daszkowski 2003). Mediale Charaktere, die diesen Idealen nahekommen, werden im Vergleich zu anderen zudem positiver und als erfolgreicher dargestellt (Fouts \& Burggraf 1999).

Studien belegen, dass ein solches Aussehen als eigenes Ideal sowie als das Ideal anderer Personen wahrgenommen und mit dem eigenen Aussehen verglichen wird (Pope et al. 2000). Dennoch wäre es falsch, aus der bloßen Existenz dieser medialen Darstellungen automatisch auf Wirkungen zu schließen. Es ist von einer Vielzahl unterschiedlicher Faktoren abhängig, ob sich die Wirkungspotenziale idealisierter Mediencharaktere auch entfalten. Relevant sind zum Beispiel Eigenschaften der Rezipienten selbst, situative und soziale Aspekte, gesellschaftliche Bedingungen, Strategien der Medienselektion, Charakteristika der genutzten Medieninhalte oder auch Nutzungsmotive (ausführlich: Baumann 2009). Konkret ist etwa das Geschlecht der Rezipienten von Bedeutung: Frauen führen in stärkerem Maße Vergleiche mit Mediencharakteren bezüglich des Aussehens durch und scheinen anfälliger für negative Wirkungen zu sein (z. B. Jones 2001). Auch 
der Grad der Auseinandersetzung mit dem eigenen Körper sowie die Zufriedenheit mit dem Aussehen und das Selbstbewusstsein gelten als wichtige moderierende Variablen (z. B. David \& Johnson 1998).

Neben Versuchen zur Bestimmung relevanter Einflussvariablen finden sich theoriebasierte Erklärungen für etwaige Wirkungen idealisierter Medienbilder: Der Kultivierungshypothese (Gerbner et al. 2002) zufolge sollte die dauerhafte mediale Darstellung idealisierter Körperbilder dazu führen, dass diese von Rezipienten als auch in der Realität allgegenwärtig und erstrebenswert angesehen werden (z. B. Rossmann \& Brosius 2005). Ein zweiter Ansatz ist die Theorie des sozialen Lernens (Bandura 2002): Danach werden attraktive Rollenbilder - wie etwa schlanke Frauen und muskulöse Männer - gelernt. In der Folge wird versucht, sie zu adaptieren (z. B. Harrison \& Cantor 1997). Die Theorie sozialer Vergleichsprozesse (Festinger 1954) als dritter Ansatz geht davon aus, dass sich Menschen mit anderen Menschen vergleichen; also vergleichen auch Rezipienten ihr Aussehen mit dem der Personen in den Medien. Die Vergleiche fallen häufig schlecht aus, da diese Personen oft für attraktiver gehalten werden. Dies kann - zumindest bei Teilen des Publikums - zu Unzufriedenheit führen (z. B. Botta 1999). Zudem besteht die Möglichkeit, dass durch die Betrachtung attraktiver Medienakteure Wunschbilder von der eigenen Person oder Vorstellungen, wie man aus Sicht anderer Personen aussehen sollte, salient werden. Im Sinne der Self-Discrepancy-Theory kann dies zu einer intensivierten und negativ erlebten Wahrnehmung einer Diskrepanz zwischen Wunsch und Wirklichkeit führen (Harrison 2001).

Diesen Ansätzen steht jedoch eine Beobachtung gegenüber: Vielen Rezipienten ist bewusst, dass die Personen in den Medien kein getreues Abbild des durchschnittlichen Aussehens der Bevölkerung abgeben und es vermessen sowie nicht erstrebenswert wäre, ihnen nachzueifern. In einer Studie von Milkie (1999) kritisierten Frauen sogar, wie Frauen in den Medien gezeigt werden. Allerdings sahen sie dennoch einen gewissen Druck, den diese Darstellungen auf sie selbst ausübten: Sie glaubten nämlich daran, dass andere - vor allem Männer - sie selbst im Vergleich zu Frauen etwa in der Werbung beurteilen würden. Wichtig wäre demzufolge weniger die eigene Sicht auf die idealisierten Schönheitsbilder, sondern die Erwartung, wie andere Menschen diese Bilder wahrnehmen. Dies führt zum Third-Person-Effekt als weiterem Ansatz, dem im Zusammenhang mit möglichen Gründen einer Wirkung medialer Frauen- und Männerdarstellungen Relevanz zugewiesen werden kann.

\subsection{Der Third-Person-Effekt}

Der Third-Person-Effekt besteht aus zwei zentralen Annahmen (Davison 1983): 1. Individuen sind der Auffassung, dass andere stärker als sie selbst von Medien beeinflusst werden - und dabei die anonyme Allgemeinheit, die dritten Personen, noch mehr als Personen aus dem unmittelbaren Umfeld (Wahrnehmungskomponente/Third-PersonPerception). 2. Diese Wahrnehmungsdifferenz führt zu Änderungen von Vor- und Einstellungen und/oder Verhaltensweisen (Verhaltenskomponente/Third-Person-Behavior). Aus Sicht des mit dem Third-Person-Effekt eng verwandten Influence-of-Presumed-Media-Influence-Approach (Gunther \& Storey 2003) führen dagegen nicht die Wahrnehmungsdifferenzen zu Konsequenzen. Vielmehr wird unterstellt, dass es Folgen haben kann, wenn Individuen den Medien einen Einfluss auf andere Rezipienten zu-

schreiben. Diesem Verständnis wird auch in einigen Studien zum Third-Person-Effekt gefolgt (z. B. Chia et al. 2004).

Es liegen inzwischen viele Studien zum Third-Person-Effekt vor, denen sich entnehmen lässt, welche medialen Einflüsse warum auf wen wahrgenommen werden und 
welche Konsequenzen sich daraus ergeben (im Überblick: Gunther et al. 2007, Huck \& Brosius 2007, Tal-Or et al. 2009). Die erste zentrale Annahme zur Wabrnehmungskomponente kann für etliche Anwendungsfelder als bestätigt gelten (Sun et al. 2008). Die Stärke der Wahrnehmungsdifferenzen ist dabei von Moderatorvariablen abhängig: Je unerwünschter die mediale Botschaft und je anfälliger die Empfänger aus Sicht der Befragten, desto größer die Third-Person-Perception. Auch die vermutete soziale Distanz zum Publikum ist wichtig: Je unähnlicher andere Personen im Vergleich zur eigenen Person empfunden werden, desto größer die Wahrnehmungsdifferenz (oder desto stärker die Vermutung, andere seien beeinflussbar; z. B. Meirick 2005). Dass andere Personen als der eigenen Person unähnlich empfunden werden, kann viele Gründe haben (McCroskey et al 2006): etwa, weil ihnen andere Eigenschaften unterstellt werden, oder auch, weil sie dem anderen Geschlecht angehören.

Die Ergebnisse von Studien zu den Folgen von Einflusswabrnebmungen sind weniger einheitlich. In einigen Untersuchungen, in denen die Wahrnehmungsdifferenzen als unabhängige Variable dienten, fanden sich signifikante Konsequenzen, in anderen indes nicht (Xu \& Gunzenbach 2008). In Studien, die ausschließlich den wabrgenommenen Einfluss auf andere als Prädiktor heranzogen, konnten dagegen die Hypothesen zu Folgen der Einflusswahrnehmung oft bestätigt werden (z. B. Gunther \& Storey 2003, Gunther et al. 2006).

\subsection{Third-Person-Effekte und medial vermittelte Schönheitsideale}

Zur Wahrnehmung medial vermittelter Schönheitsideale liegen mehrere Studien vor. Häufig wurden dabei Wahrnehmungsdifferenzen gemessen (Chia 2007, 2009; Choi et al. 2008, David \& Johnson 1998, David et al. 2002). Nur vereinzelt wurden zusätzlich Folgen dieser Differenzen analysiert (Chia 2007). In einigen Studien galt dagegen die Konzentration allein der Frage, welche Folgen die Vermutungen über die Wahrnehmungen anderer Personen haben (Park 2005, Thomsen 2002). Es wurden durchgängig ausschließlich Studentinnen, also Frauen in jungem Alter, befragt.

In der Mehrheit der Studien wurden den Befragten Bilder weiblicher Fotomodelle zumeist aus Werbeanzeigen - vorgelegt (in anderen Studien wurde auf Stimuli verzichtet). Die Bilder wurden zum Teil systematisch variiert: In einer Untersuchung (Chia 2007, 2009) wurden asiatischen Studentinnen Bilder von asiatischen und „caucasian“ („light skinned humans of European origin“; Chia 2009, S. 425) Frauen gezeigt. David et al. (2002) befragten weiße und dunkelhäutige Studentinnen und präsentierten ihnen Bilder mit weißen und dunkelhäutigen Models. Auf diese Weise waren unterschiedliche Vergleiche möglich.

Zusätzliche Differenzierungen ergaben sich unter anderem durch die Auswahl der Personengruppen, deren Beeinflussung von den Befragten eingeschätzt werden sollte: David et al. (2002) ermittelten etwa den vermuteten Einfluss auf weiße und auf dunkelhäutige Frauen, Chia $(2007,2009)$ den vermuteten Einfluss auf weibliche und männliche Freunde, Choi et al. (2008) den vermuteten Einfluss auf verschiedene Gruppen von Frauen und Männern (Freund/inn/e/n, Student/inn/en an eigener Universität sowie in den USA).

Ergebnisse zur Wabrnebmung von Einflüssen: In den Studien bestätigte sich die generelle Annahme, dass andere Personen als empfänglicher für Wirkungen einer Rezeption von Bildern attraktiver Frauen gehalten werden als die eigene Person. Dies gilt für vermutete Einflüsse auf Vorstellungen vom idealen Körpergewicht, auf die Zufriedenheit mit dem eigenen Aussehen oder auf die Entwicklung von Essstörungen (z. B. David \& Johnson 1998). Häufig stieg auch mit zunehmender Distanz zu den anderen Perso- 
nengruppen die Differenz zwischen dem wahrgenommenem Einfluss auf sich selbst und andere an: So wurden etwa Freundinnen, also die nähere Umgebung, für nicht so beeinflussbar gehalten wie weiter entfernte Personengruppen (Choi et al. 2008).

Es sind jedoch Differenzierungen zu beachten. Sie gelten zunächst für vermutete Wirkungen auf die eigene Person: David et al. (2002) gingen davon aus, dass sich die Befragten jeweils für beeinflussbarer durch die Models der eigenen Hautfarbe halten würden. Bei dunkelhäutigen Teilnehmerinnen war dies der Fall, die weißen Probandinnen bekundeten indes gleich starke Einflüsse durch weiße und dunkelhäutige Models auf sich selbst.

Auch bei den vermuteten Wirkungen auf andere Personen bzw. den Wabrnebmungsdifferenzen stechen einige Resultate hervor. Relevant für die vorliegende Studie sind primär Ergebnisse zu geschlechtsspezifischen Unterschieden: In den Untersuchungen von Chia $(2007,2009)$ sowie Choi et al. (2008) vermuteten die weiblichen Befragten, ihre männlichen Freunde seien durch die idealisierten Frauenbilder beeinflussbarer als sie selbst und als ihre weiblichen Freunde. Bei anderen vergleichenden Einschätzungen, etwa zur Beeinflussbarkeit weiblicher und männlicher Studierender der eigenen Universität, ergaben sich allerdings kaum Unterschiede (Choi et al. 2008). Gerade in den Studien von Chia $(2007,2009)$ und Choi et al. (2008) wurde jedoch nur nach vermuteten Einflüssen auf Einstellungen zum idealen Aussehen von Frauen gefragt. Vermutete Einflüsse zum Beispiel auf die Zufriedenheit der unterschiedlichen Personengruppen mit ihrem eigenen Aussehen wurden nicht thematisiert und entsprechend nicht mit Blick auf andere Frauen und Männer analysiert.

Ergebnisse zu den Folgen der Einflusswabrnebmungen: Thomsen (2002) konnte belegen, dass das Lesen von Frauenzeitschriften einen direkten Einfluss darauf hatte, das eigene Aussehen kritisch zu betrachten. Etwas schwächer, aber ebenfalls signifikant waren zudem indirekte Einflüsse auf diese Variable: Sie ergaben sich über die Ansicht der Probandinnen, die Vorstellungen von Männern zum idealen Aussehen von Frauen seien durch die mediale Darstellung beeinflussbar (siehe auch Milkie 1999). In einer Untersuchung von Park (2005) war der direkte Einfluss der Mediennutzung auf den Wunsch, schlank zu sein, eher gering. Aussagekräftiger waren indirekte Effekte, bei denen der Glaube, andere Männer und vor allem andere Frauen würden in ihren Schönheitsidealen medial beeinflusst, ein Teil der Wirkungskette war. Dies wird als Folge normativer Einflüsse gesehen: Medien legen Verhaltensweisen nahe, die von anderen - so die Vermutung des Einzelnen - angenommen werden. Um nicht abzuweichen, wird versucht sich anzupassen (Tal-Or et al. 2009).

Chia (2007) kam dagegen zu anderen Befunden. Sie prüfte, ob der wahrgenommene Einfluss einer Rezeption von Modelbildern Auswirkungen auf eigene Vorhaben zur Gewichtsreduktion hatte. Es zeigten sich fast ausnahmslos signifikante Einflüsse der Selbstwahrnehmung: Je größer der wahrgenommene Einfluss der Bilder auf sich selbst, desto stärker die Bereitschaft zu gewichtsreduzierendem Verhalten. Die vermuteten Einflüsse auf weibliche wie männliche Freunde waren dagegen nahezu bedeutungslos.

Insgesamt zeigt der Forschungsstand mit Blick auf die Wahrnehmungsdifferenzen, dass der Einfluss einer Rezeption attraktiver Medienakteure auf andere stärker eingeschätzt wird als auf die eigene Person. Wenn Models der eigenen Person ähnlich sind (im überprüften Fall: gleiche Hautfarbe), dann ist die selbst zugeschriebene Wirkung zumindest partiell größer. In Studien, die wahrgenommene Einflüsse auf andere Frauen und Männer getrennt erfassten, wurden Männer überwiegend als beeinflussbarer durch Bilder weiblicher Models gehalten als Frauen - betont werden sollte jedoch, dass dort vermutete Einflüsse auf Schönheitsideale (bzw. konkret Schlankheitsideale) gemessen 
wurden, und nicht etwa vermutete Einflüsse auf die Zufriedenheit mit dem eigenen Aussehen. Zu den individuellen Folgen der Wahrnehmung von Einflüssen liegen bislang nur wenige Befunde vor: Zum Teil ergeben sich Folgen aufgrund von Einflusszuschreibungen auf die eigene Person, zum Teil aufgrund der Wahrnehmung von Einflüssen auf andere Personen.

Die Erkenntnisse beruhen auf einer geringen Zahl von Studien. Zudem fällt auf, dass nicht in einer einzigen Untersuchung Männer befragt wurden. Auch die vermuteten Wirkungen medial dargestellter Männer wurden noch nicht im Kontext des Third-Person-Effekts geprüft. Auf diese Lücke weisen auch Choi et al. (2008, S. 161) hin: „(..) future research should explore men's third-person perceptions when they are exposed to women's idealized body image ads. In addition, how women view men's' (sic!) idealized body image will also provide a richer picture to better understand the role of gendered others in the idealized body image domain“. Diese Punkte wurden daher in der vorliegenden Untersuchung aufgegriffen.

\subsection{Hypothesen}

Die ersten Hypothesen beziehen sich auf die Wabrnebmung und Wabrnebmungsdifferenzen. Überprüft werden jeweils Einschätzungen zum Einfluss auf die Zufriedenheit mit dem Aussehen. Diese Variable ist für die Forschung zu Wirkungen idealisierter Medienbilder noch zentraler als die in einigen Third-Person-Studien (z. B. Chia 2007) abgefragte Einschätzung, in welchem Ausmaß allgemeine Schönheitsideale beeinflusst werden.

Zunächst wird angenommen, dass anderen Personen ein größerer Einfluss unterstellt wird als der eigenen Person - und dabei der Allgemeinheit ein noch stärkerer Einfluss als Personen der unmittelbaren Umgebung (Davison 1983). Die Annahme wurde zweigeteilt, um mögliche Wahrnehmungsdifferenzen zunächst getrennt nach Geschlechtern zu untersuchen:

H1a: Frawen werden den Effekt der Frauenbilder auf ibre weiblichen Freunde/Bekannten höher einschätzen als auf sich selbst und Frauen im Allgemeinen eine noch gröBere Beeinflussung unterstellen als ibren weiblichen Freunde/Bekannten.

H1b: Männer werden den Effekt der Männerbilder auf ibre männlichen Freunde/ Bekannten höher einschätzen als auf sich selbst und Männern im Allgemeinen eine noch größere Beeinflussung unterstellen als ibren männlichen Freunde/Bekannten.

Die zweite Hypothese berücksichtigt Geschlechtsunterschiede, beschränkt sich aber auf Aussagen zur eigenen Person. In Anlehnung an David et al. (2002) - die allerdings nach der Hautfarbe differenzierten - wird angenommen, dass ein stärkerer Einfluss auf die eigene Person eingeschätzt wird, wenn die Models eine größere Ähnlichkeit zur eigenen Person aufweisen. Entsprechend gilt, dass Frauen sich selbst stärker von Frauenbildern beeinflusst sehen sollten, Männer dagegen von Männerbildern:

H2: Effekte auf die eigene Person werden als größer angeseben, wenn auf den Bildern Models des eigenen Geschlechts abgebildet sind.

Mit der dritten Hypothese werden Wirkungen auf andere Personen bedacht. Im Anschluss an die bisherige Forschung sollen vermutete Wirkungen medialer Idealbilder des eigenen Geschlechts auf Personen des eigenen und des jeweils anderen Geschlechts überprüft werden. Laut Befragungen von Frauen (Chia 2007, 2009; Choi et al. 2008) werden Männer mehr als Frauen in ihren Ansichten zu Schönheitsidealen für beeinflussbar durch Bilder weiblicher Models gehalten. Dies deckt sich mit den Erkenntnissen der Third-Person-Forschung zur Rolle der sozialen Distanz. Genauso müssten männliche Befragte eher andere Frauen als andere Männer für beeinflussbar durch Männer- 
bilder halten. In dieser Studie werden jedoch nicht vermutete Einflüsse auf allgemeine Schönheitsideale erfragt, sondern Einschätzungen zu den Auswirkungen auf die Zufriedenheit mit dem eigenen Aussehen. In diesem Fall ist zu erwarten, dass die Befragten den gleichgeschlechtlichen Bildern eine höhere Wirkung auf Personen des gleichen Geschlechts unterstellen auf als Personen anderen Geschlechts:

H3a: Franen werden den Effekt von Frauenbildern auf andere Franen höber einschätzen als den Effekt von Frauenbildern auf andere Männer.

H3b: Männer werden den Effekt von Männerbildern auf andere Männer höber einschätzen als den Effekt von Männerbildern auf andere Frauen.

Die vierte Hypothese gilt möglichen Folgen der Einflusszuschreibungen. Postuliert wird, dass die zugeschriebenen Einflüsse wiederum Auswirkungen haben - als abhängige Variable diente die Bereitschaft zur Veränderung des eigenen Aussehens. Chia (2007) zufolge hatte der wahrgenommene Einfluss idealisierter Medienbilder auf die eigene Person Folgen; laut Park (2005) und Thomsen (2002) hatte dagegen der wahrgenommene Einfluss auf andere Franen und Männer Auswirkungen. Daher wurden für beide Möglichkeiten Annahmen formuliert:

H4a: Je stärker der wabrgenommene Effekt gleichgeschlechtlicher Models auf die eigene Person, desto größer ist die Bereitschaft, sich um eine Verbesserung des eigenen Aussebens zu bemühen.

H4b: Je stärker die wabrgenommenen Effekte gleichgeschlechtlicher Models auf andere Personen, desto größer ist die Bereitschaft, sich um eine Verbesserung des eigenen Aussebens zu bemühen.

\section{Methode}

\subsection{Ablauf der Studie}

Die Hypothesen wurden in einer experimentell angelegten Befragung getestet. Frauen und Männern wurden dort entweder Bilder weiblicher oder männlicher Models präsentiert.

Die Studie wurde als Online-Befragung durchgeführt. Dies hatte forschungsökonomische Gründe, da über E-Mail-Verteiler und Internetforen vergleichsweise einfach und ohne Kosten viele Personen zur Teilnahme eingeladen werden konnten. Außerdem konnten - anders als etwa bei telefonischen Befragungen - die digitalisierten Modelbilder (siehe Kap. 3.2) in das Online-Instrument eingebaut und systematisch variiert präsentiert werden. Ein zusätzlicher Vorteil ist die Abwesenheit eines Interviewers. Dadurch erhöhen sich die subjektive empfundene Selbstbestimmtheit und die Anonymität (Taddicken 2008), was wiederum zu einer Verringerung sozial erwünschten Antwortverhaltens führen kann.

Ein wesentlicher Nachteil von Online-Befragungen ist, dass bei einer Einladung über E-Mail-Verteiler oder Internetforen nur wenig Einfluss auf die Zusammensetzung der Stichprobe genommen werden kann. Dies musste auch in der vorliegenden Studie in Kauf genommen werden. Daher galt es vorrangig, unter Berücksichtigung der Vorannahmen und der Ziele der Studie, ausreichend Frauen und Männer zu rekrutieren. Um eine Vergleichbarkeit mit den Vorgängerstudien - in denen stets Studentinnen befragt wurden - zu gewährleisten, sollten zudem primär jüngere Personen zur Teilnahme bewogen werden.

Konkret wurden die Befragten über studentische E-Mail-Verteiler oder Internetforen auf themenspezifischen Seiten (z. B. www.gofeminin.de; http://forum.menslabel.de/ 
forum.php) zu einer Studie über „Schönheitsideale in den Medien“ eingeladen. ${ }^{1}$ Damit wurde das (ohnehin schnell erkennbar werdende) Thema der Untersuchung direkt und offen angesprochen, ohne im Detail auf spezifische Annahmen und Hintergründe einzugehen.

Der Fragebogen enthielt zunächst einführende Items zur Mediennutzung. Danach wurden vier Bilder vorgeführt - jeweils ein Bild pro Seite des Online-Fragebogens. Es wurde zufällig ausgewählt, ob es sich um vier Frauen- oder vier Männerbilder handelte. ${ }^{2}$ Das Geschlecht der präsentierten Models diente somit als experimenteller Faktor mit zwei Ausprägungen. Direkt nach Betrachtung der Bilder musste das Aussehen der Models beurteilt werden.

In der Folge mussten weitere Statements zu den Bildern abgegeben werden. Zentral waren Aussagen, anhand derer Vermutungen zu Auswirkungen dieser Bilder auf die Zufriedenheit mit dem Aussehen abgegeben werden sollten. Dabei schätzten die Befragten Auswirkungen auf sich selbst ein und gaben zusätzlich Vermutungen zu den Auswirkungen der Bilder auf die Zufriedenheit bei weiblichen Freunden, männlichen Freunden, Frauen im Allgemeinen und Männern im Allgemeinen ab. Diese Items - sowie alle folgenden Items - wurden so formuliert, dass sie für alle Befragten (Frauen und Männer) sowie für beide Varianten der experimentell manipulierten Variable (Frauenund Männerbilder) identisch waren.

Es ergab sich somit das in Abbildung 1 dargestellte Muster. Die unterschiedlichen Fallzahlen für die Einschätzung der Frauen- bzw. Männerbilder durch die Teilnehmerinnen entstanden dadurch, dass Frauen die Befragung oft abbrachen, wenn Männerbilder präsentiert wurden.

\section{Abbildung 1: Untersuchungsplan}

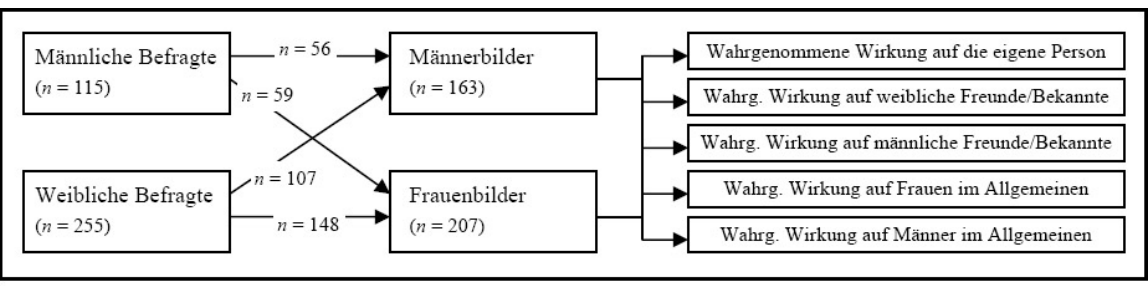

Im Anschluss an die Items zu den Einflusswahrnehmungen mussten weitere Items beantwortet werden. Sie bezogen sich unter anderem auf die Beurteilung des eigenen Aussehens sowie auf potenzielle Wege einer Veränderung dieses Aussehens.

\subsection{Bildmaterial und Manipulation Check}

Die Präsentation des Stimulusmaterials erfolgte in Anlehnung an vorherige Studien (David et al. 2002; Chia 2007, 2009; Choi et al. 2008). Den Befragten wurden vier Bilder von

1 Einladungen in Foren zu völlig anderen Themen als dem der Studie erwiesen sich nicht als sinnvoll.

2 Überlegungen, den Befragten Frauen- und Männerbilder zu zeigen und sie Einschätzungen zu deren Wirkungen abgeben zu lassen, wurden - auch als Folge vorheriger Tests - verworfen. Vor allem wegen der vielen und recht ähnlichen Third-Person-Items (siehe Kap. 3.3) wurde die Befragung dann als zu lang und monoton empfunden. 
Frauen oder von Männern gezeigt. Den Bildern waren die folgenden Sätze vorangestellt: „Wie Sie wahrscheinlich wissen, werden in den Medien sehr häufig Menschen dargestellt, die den gängigen Schönheitsidealen entsprechen. (...) Sie sehen auf den folgenden Seiten einige Fotos von [weiblichen] [männlichen] Models, wie Sie sie vielleicht aus Anzeigenkampagnen kennen. Sie sind aus Zeitschriften entnommen. Bitte schauen Sie sich die Bilder an." Die Bilder stammten zumeist aus Anzeigen. Sie lagen digitalisiert vor und konnten so in den Online-Fragebogen integriert werden. Hinweise, etwa auf Produktmarken, wurden entfernt. Es wurde darauf geachtet, dass die vier Frauen- und die vier Männerbilder jeweils ähnlich waren: Alle Models waren vom Kopf bis zur Hüfte bzw. bis zu den Knien zu sehen. Die Frauen trugen Bikini oder Unterwäsche, die Männer Jeans oder Shorts bei freiem Oberkörper. Die Frauen waren schlank, die Männer muskulös.

Das Aussehen der präsentierten Frauen und Männer musste von den Befragungsteilnehmern beurteilt werden. Dieses Vorgehen diente als Manipulation Check. Dazu wurden vier Items formuliert. Sie waren in der Frauen- und der Männerbilder-Version fast identisch. Anhand von zwei Items sollten die Befragten die Attraktivität der Models einschätzen; mit zwei Items sollten sie angegeben, wie deren Beurteilung durch andere Menschen vermutet wurde. Die Items ließen sich jeweils zusammenfassen (Cronbachs Alpha, auch bei getrennter Berechnung für Teilnehmerinnen und Teilnehmer, zwischen .68 und .71). Die Models wurden als attraktiv eingeschätzt (5-stufige Skalen; hohe Werte weisen auf große Attraktivität hin). Frauen und Männer unterschieden sich nur sehr geringfügig in ihrer Bewertung der abgebildeten Frauen (weibliche Teilnehmer: $M=4.51, S D=.51$; männliche Teilnehmer: $M=4.42, S D=.55 ; t(201)=-1.02, n s)$ und Männer (weibliche Teilnehmer: $M=4.21, S D=.63$; männliche Teilnehmer: $M=4.07$, $S D=.57 ; t(160)=-1.30, n s)$. Allerdings zeigen die Daten auch, dass die abgebildeten Frauen insgesamt für etwas attraktiver gehalten wurden als die Männer. Dies muss bei der Interpretation der weiteren Ergebnisse berücksichtigt werden.

\subsection{Messungen der Konstrukte}

Wabrnehmungsdifferenz: Zur Messung des wahrgenommenen Einflusses der zuvor gezeigten Bilder wurden zwei Basisitems formuliert. Mit einem Item wurden die vermuteten Wirkungen der gezeigten Bilder auf die Zufriedenheit mit dem Ausseben erfasst. Mit dem anderen Item sollte eingeschätzt werden, inwiefern die Bilder den Wunsch nach einer Veränderung von Körper und Aussehen beeinflussten. Beide Basisitems wurden in fünf Versionen abgefragt: Wahrnehmung des Einflusses auf die eigene Person, auf weibliche Freunde und Bekannte, auf männliche Freunde und Bekannte, auf Frauen im Allgemeinen sowie auf Männer im Allgemeinen (alle Items: 5-stufige Skala mit $1=$ „trifft überhaupt nicht zu“ bis $5=$ „trifft voll und ganz zu“). In allen fünf Versionen konnten die beiden Basisitems zusammenfasst werden (die Cronbachs-Alpha-Werte für die jeweils zwei Items lagen, auch bei getrennter Rechnung für Frauen und Männer, zwischen .72 und .83). Somit ergab sich eine Gesamtvariable zur Einflusswahrnehmung, die für fünf Personengruppen formuliert war.

Folgen der Einflusswabrnehmung: Es wurden drei Handlungen bzw. Handlungsabsichten gemessen (alle Items: $1=$ „trifft überhaupt nicht zu“ bis $5=$,trifft voll und ganz

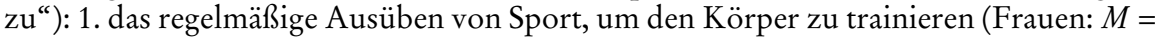
2.86, $S D=1.31$; Männer: $M=2.88, S D=1.24)$; 2 . das Einhalten bestimmter Ernährungsformen, z. B. einer Diät, um eine gute Figur zu haben (Frauen: $M=2.43, S D=1.30$; Männer: $M=1.80, S D=1.19) ; 3$. die Überlegung, eine Schönheitsoperation durchführen zu lassen (Frauen: $M=1.99, S D=1.35$; Männer: $M=1.34, S D=.92$ ). Mit ihrer Hilfe 
sollten mögliche Folgen einer vermuteten Wirkung idealisierter Medienbilder für die eigene Person erfasst werden. Aufgrund schlechter Cronbachs-Alpha-Werte war eine Zusammenfassung der Items nicht angezeigt. Der Unterschied der Werte war zwischen den Befragten, die die Frauenbilder gesehen hatten, und den Befragten, die die Männerbilder gesehen hatten, stets nicht signifikant - dies gilt für Frauen wie für Männer.

Weitere Variablen: Andere Variablen hatten kontrollierenden Charakter - etwa die Beurteilung des eigenen Aussehens, gemessen mit Items aus einer Skala von Strauß und Appelt (1983): Zum einen wurde die Zufriedenheit mit dem Aussehen erfasst (Frauen: $M=3.37, S D=.94$; Männer: $M=3.56, S D=.84$ ), zum anderen die Sensibilität bezüglich des Aussehens (Frauen: $M=3.85, S D=.79$; Männer: $M=3.29, S D=.89$; jeweils 5-stufige Skalen; hohe Werte = hohe Zufriedenheit/starke Sensibilität). Zwischen den Versionen (Frauen- vs. Männerbilder) gab es keine signifikanten Unterschiede in den Angaben von Frauen und Männern.

Zusätzlich erhoben wurden Einstellungen zur Präsentation von Frauen und Männern in den Medien, spezielle Aspekte der Mediennutzung (z. B. Interesse an Berichten über Prominente sowie „Klatsch und Tratsch“), Items zur Erfassung der eigenen Beeinflussbarkeit sowie der Beeinflussbarkeit der allgemeinen Bevölkerung und Angaben zur Soziodemografie.

\subsection{Stichprobe}

Die Studie basiert auf insgesamt 370 gültigen Fällen. Ungefähr zwei Drittel der Befragten waren Frauen $(n=255)$, ein Drittel Männer $(n=115$; siehe Abbildung 1). Es wurden nur Personen berücksichtigt, die 30 Jahre und jünger waren. Ältere Personen wurden ausgeschlossen, um eine möglichst homogene Stichprobe zu erhalten. ${ }^{3}$ Zudem sollte die Stichprobe vom Altersschnitt her mit anderen Studien zum Thema vergleichbar sein. Ferner schien es sinnvoll zu sein, dass das Alter der Befragten in etwa mit dem der gezeigten Models übereinstimmte. Das Durchschnittalter der Befragten betrug 22.13 Jahre $(S D=3.17$ Jahre; Frauen: $M=21.72, S D=3.11$; Männer: $M=23.04, S D=3.14) .87,8$ Prozent hatten als höchsten Bildungsgrad das Abitur oder einen Hochschulabschluss (Frauen: 85,9\%; Männer: 92,2 \%). Dieser hohe Bildungsgrad ist auffällig, korrespondiert aber ebenfalls mit dem der in den vorhergehenden Untersuchungen befragten Frauen. Im Vergleich der Treatmentgruppen (präsentierte Frauen- vs. präsentierte Männerbilder) ergaben sich keine deutlichen Unterschiede für Alter und Bildungsgrad (Frauenbilder: $M=21.99, S D=3.04 ; 88,9 \%$ mit Abitur oder Hochschulabschluss; Männerbilder: $M=22.32, S D=3.33 ; 86,5 \%$ mit Abitur oder Hochschulabschluss). Insgesamt ist anhand der Stichprobe erkennbar, dass durch die Studie in erster Linie Aussagen für jüngere und höher gebildete Personen getroffen werden können.

\section{Ergebnisse}

Die Hypothesen 1 bis 3 beziehen sich auf den vermuteten Einfluss der Bilder auf die eigene Person und andere Personengruppen. Basis der Berechnungen sind die Antworten zu den Items, mit denen der Einfluss der Frauen- oder Männerbilder auf die verschiedenen Personen(gruppen) eingeschätzt wurde. Die deskriptiven Ergebnisse finden sich in Tabelle 1.

3 Dadurch wurden 22 gültige Fälle ausgeschlossen. Probehalber durchgeführte Analysen ergaben, dass dies keine erwähnenswerten Änderungen der Studienergebnisse nach sich zog. 
Tabelle 1: Items zur Messung der Einflusswabrnehmungen

\begin{tabular}{|c|c|c|c|c|c|c|}
\hline & \multicolumn{5}{|c|}{ Zugeschriebene Wirkung ... } \\
\hline & & $\begin{array}{l}\ldots \text { auf die } \\
\text { eigene Person }\end{array}$ & $\begin{array}{l}\ldots \text { auf weib- } \\
\text { liche Freunde } \\
\text { und Bekannte }\end{array}$ & $\begin{array}{l}\text {... auf männ- } \\
\text { liche Freunde } \\
\text { und Bekannte }\end{array}$ & $\begin{array}{c}\ldots \text { auf } \\
\text { Frauen im } \\
\text { Allgemeinen }\end{array}$ & $\begin{array}{c}\ldots \text { auf } \\
\text { Männer im } \\
\text { Allgemeinen }\end{array}$ \\
\hline \multirow{2}{*}{$\begin{array}{l}\text { Weibliche } \\
\text { Befragte }\end{array}$} & $\begin{array}{l}\text { Frauenbilder } \\
n=144-147\end{array}$ & $\Gamma 3.69(1.11)$ & $3.81(.86) \underline{\mathrm{H} 3}$ & $\mathrm{H1} 2.39(.92)$ & $4.13(.71)$ & H3 $2.49(.95)$ \\
\hline & \begin{tabular}{|l} 
Männerbilder \\
$n=102-105$
\end{tabular} & L ${ }^{\mathrm{H} 2} 2.93(1.32)$ & $3.15(1.01)$ & $3.45(.81)$ & $3.51(.88)$ & $3.76(.68)$ \\
\hline \multirow{2}{*}{$\begin{array}{l}\text { Männliche } \\
\text { Befragte }\end{array}$} & $\begin{array}{l}\text { Frauenbilder } \\
n=55-57\end{array}$ & $\Gamma_{\mathrm{H} 2} 2.23(1.27)$ & $3.57(.83)$ & $2.43(.92)$ & $4.00(.62)$ & $2.65(.95)$ \\
\hline & $\begin{array}{l}\text { Männerbilder } \\
n=55-56\end{array}$ & L $2.89(.96)$ & $2.71(.86) \stackrel{\mathrm{H3}}{-}$ & $-2.95(.77)$ & $3.04(.98)-$ & H3 $3.44(.70)$ \\
\hline
\end{tabular}

Mittelwerte (Standardabweichungen); Ausprägungen aller Items von 1 bis 5; wobei $1=$ Zuschreibung eines sehr geringen Einflusses; $5=$ Zuschreibung eines sehr großen Einflusses

Hypothesen 1a/1b: Die erste Hypothese wurde nach den Geschlechtern getrennt. Postuliert wurde, dass Frauen sich selbst am wenigsten durch die Frauenbilder beeinflusst sahen, ihre weiblichen Freunde und Bekannten dagegen etwas stärker, am stärksten jedoch Frauen im Allgemeinen (H1a). Männliche Befragte sollten ihre männlichen Freunde und Bekannten im Vergleich zu sich selbst als beeinflussbarer durch Männerbilder einschätzen, am stärksten indes Männer im Allgemeinen (H1b). Die Mittelwerte sind in Tabelle 1 erkennbar.

Die Hypothesen wurden per Varianzanalysen mit Messwiederholungen geprüft, die einzelnen Mittelwerte zudem einem Post-Hoc-Vergleich mit Bonferroni-Korrektur unterzogen. Zusätzlich wurde die Beurteilung des eigenen Aussehens als Kovariate berücksichtigt. Für Hypothese 1a und 1b ergeben sich nahezu identische Ergebnisse: Bei den befragten Frauen zeigen sich in der Varianzanalyse signifikante Gesamteffekte $\left(F(1.72,140)=7.04, p<.01 ; \eta^{2}=0.05\right)$; gleiches gilt für die Männer $(F(1.73,55)=6.09$, $\left.p<.01 ; \eta^{2}=0.10\right) .{ }^{4}$ Dies spricht für die Hypothesen. Die Post-Hoc-Analysen belegen jedoch, dass bei den befragten Frauen und Männern nur die jeweiligen Unterschiede in den Werten 1. zwischen eigener Person und gleichgeschlechtlichen Frauen/Männern im Allgemeinen sowie 2. zwischen weiblichen/männlichen Freunden/Bekannten und gleichgeschlechtlichen Frauen/Männern im Allgemeinen signifikant sind - zwischen erster Person und gleichgeschlechtlichen Freunden/Bekannten jedoch nicht. Die Hypothesen $1 \mathrm{a}$ und $1 \mathrm{~b}$ werden daher nur partiell bestätigt. Eindeutig hypothesenkonform sind aber die Unterschiede zwischen erster Person und dritten Personen.

Hypothese 2: Laut Hypothese 2 sollten die Befragten die Wirkungen der Bilder auf sich selbst als stärker einschätzen, wenn dort Personen des eigenen Geschlechts abgebildet waren. Zur Überprüfung wurde analysiert, ob die Werte für die der eigenen Person zugeschriebenen Einflüsse bei den weiblichen Befragten, denen die Frauenbilder gezeigt wurden, höher waren als bei weiblichen Befragten, die Einflüsse der Männerbilder einschätzen sollten. Eine univariate Varianzanalyse - bei der ebenfalls die Beurteilung des eigenen Aussehens als Kovariate berücksichtigt wurde - bestätigt dies $(F(1,250)=23.19$, $\left.p<.001 ; \eta^{2}=0.09\right)$. Gleiches gilt für die befragten Männer: Sollten sie den Einfluss von Männerbildern auf sich selbst einschätzen, dann waren die Werte höher als bei den

4 Wegen ungleicher Varianzen zwischen den Items wurden Greenhouse-Geisser-Korrekturen vorgenommen. 
selbstzugeschriebenen Einflüssen durch Frauenbilder $\left(F(1,112)=7.46, p<.01 ; \eta^{2}=0.06\right.$; für deskriptive Werte siehe jeweils Tabelle 1). Damit kann Hypothese 2 bestätigt werden.

Hypothesen 3a/3b: Die Hypothesen 3a und 3b bezogen sich auf Wirkungen, die anderen zugeschrieben werden. Mit Blick auf die Frauenbilder wurde angenommen, dass Frauen deren Effekt auf andere Frauen höher einschätzen würden als den Effekt auf andere Männer (H3a). Umgekehrt galt mit Blick auf die Männerbilder, dass Männer deren Effekt auf andere Männer höher einschätzen würden als den Effekt auf andere Frauen (H3b). Die deskriptiven Werte (siehe Tabelle 1) verdeutlichen, dass den Bildern weiblicher Models von den weiblichen Befragten eine höhere Wirkung auf andere Frauen im Vergleich zu anderen Männern zugewiesen wird. Umgekehrt wird Bildern mit männlichen Models von den männlichen Befragten eine höhere Wirkung auf andere Männer im Vergleich zu anderen Frauen unterstellt. Bei den Frauenbildern sind diese Differenzen erheblich und laut Varianzanalysen mit Messwiederholungen - inklusive der Beurteilung des eigenen Aussehens als Kovariate - signifikant (wahrg. Einfluss auf weibliche Freunde vs. wahrg. Einfluss auf männliche Freunde: $F(1,143)=47.51, p<.001$; $\eta^{2}=0.25$; wahrg. Einfluss auf Frauen im Allgemeinen vs. wahrg. Einfluss auf Männer im Allgemeinen: $\left.F(1,141)=53.00, p<.001 ; \eta^{2}=0.28\right)$. Bei den Männerbildern sind die Unterschiede jedoch eher gering und laut Varianzanalysen unter Berücksichtigung der Koviariate nicht signifikant (wahrg. Einfluss auf weibliche Freunde vs. wahrg. Einfluss auf männliche Freunde: $F(1,55)=0.20, n s ; \eta^{2}=0.00$; wahrg. Einfluss auf Frauen im Allgemeinen vs. wahrg. Einfluss auf Männer im Allgemeinen: $F(1,56)=0.26, n s ; \eta^{2}=$ 0.01). Hypothese 3 a kann damit bestätigt werden. Hypothese $3 \mathrm{~b}$, obwohl die Mittelwerte von der Tendenz her mit der Annahmen übereinstimmen, nicht.

Zur Illustration insbesondere der Resultate zu den Hypothesen $3 \mathrm{a}$ und $3 \mathrm{~b}$ wurden die bereits in Tabelle 1 dargestellten Resultate zu den Einflusswahrnehmungen grafisch aufbereitet (siehe Abbildung 2). In der Abbildung ist mit Blick auf die Werte zu den Hypothesen $3 \mathrm{a}$ und $3 \mathrm{~b}$ zu erkennen, dass sich bei den Frauenbildern ein Zick-ZackMuster ergibt - auch dann, wenn die Einflüsse der Frauenbilder von männlichen Befragten eingeschätzt wurden. Bei den Männerbildern ist dagegen ein eher leichter, kontinuierlicher Anstieg zu erkennen - auch dann, wenn die Einflüsse der Männerbilder von weiblichen Befragten eingeschätzt wurden. ${ }^{5}$

Hypothesen 4a/4b: Neben den Wahrnehmungsdifferenzen wurden mögliche Folgen der Einflusswahrnehmung geprüft. Postuliert wurde, dass die Bemühungen der Befragten um eine Verbesserung ihres Aussehens umso größer wären, je stärker der wahrgenommene Einfluss gleichgeschlechtlicher Models auf die eigene Person (H4a) und auf andere Personen (H4b) sein würde. Dazu wurden hierarchische Regressionen gerechnet: zum einen für weibliche Befragte, die Frauenbilder gesehen hatten; zum anderen für männliche Befragte, denen die Männerbilder gezeigt worden waren. Außerdem wurden die Regressionen jeweils separat für die drei Versionen der abhängigen Variable vollzogen - die Varianten waren: 1. regelmäßiger Sport, um den Körper zu trainieren, 2. Einhalten von Ernährungsformen, z. B. Diät, um eine gute Figur zu haben, 3. Überlegungen, eine Schönheitsoperation durchführen zu lassen.

Somit wurden sechs Regressionen gerechnet. Der erste Prädiktoren-Block bestand immer aus folgenden Kontrollvariablen: Bewertung der Attraktivität der Models, Zufriedenheit der Befragten mit ihrem Aussehen, Sensibilität bezüglich ihres Aussehens, Interesse an Berichten über Prominente bzw. „Klatsch und Tratsch“ (andere Variablen

5 Die Reihenfolge der verschiedenen Einflusspersonen auf der X-Achse wurde beliebig gewählt. Bei einer anderen Reihenfolge hätten sich andere Muster ergeben, was aber nichts an den Unterschieden zwischen der Wahrnehmung der Frauen- und Männerbilder geändert hätte. 
Abbildung 2: Grafische Darstellung der Ergebnisse zu den Einflusswabrnebmungen

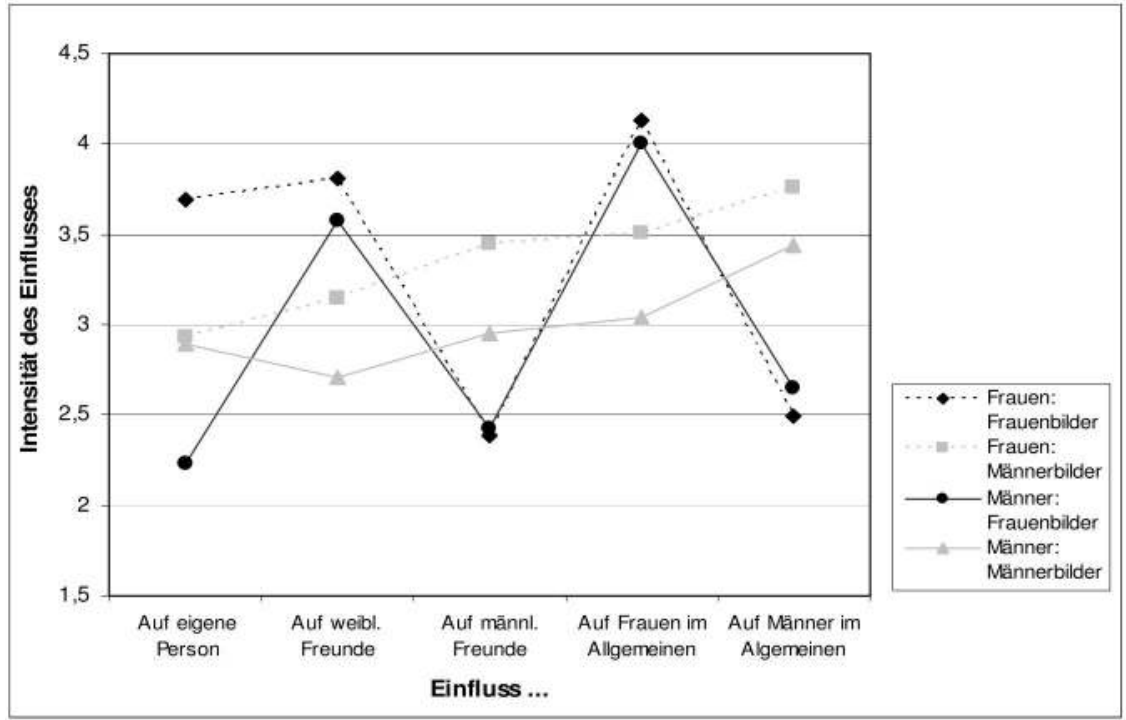

Mittelwerte; Ausprägungen aller Items von 1 bis 5 ; wobei $1=$ Zuschreibung eines sehr geringen Einflusses; 5 = Zuschreibung eines sehr großen Einflusses.

Lesebeispiel: Frauen schätzen den Einfluss der Frauenbilder auf die eigene Person insgesamt mit $\mathrm{M}=3.69$ ein (linke schwarze Raute), den Einfluss von Männerbildern auf die eigene Person jedoch mit $\mathrm{M}=2.93$ (linkes graues Quadrat)

hatten sich als nicht relevant erwiesen). Im zweiten Schritt wurden die Variable zum wahrgenommenen Einfluss auf die eigene Person (H4a) und außerdem die vier Variablen zur Messung der Einflusszuschreibungen mit Blick auf die anderen Personengruppen (H4b) berücksichtigt.

In drei der sechs Regressionen war die Wirkung der Einflusswahrnehmungen in keiner Weise signifikant - die Ergebnisse dieser Analysen werden nicht im Detail aufgeführt. Unter drei Bedingungen zeigten sich vereinzelte Effekte. Die Ergebnisse dazu finden sich in Tabelle 2. Sie werden mit Blick auf die für die Hypothesen relevanten Variablen erläutert.

Bei den befragten Frauen ergibt sich insgesamt ein signifikanter Einfluss des zweiten Blocks, in dem die Variablen zur Messung der Einflusswahrnehmungen zusammengefasst sind, auf die Diätbereitschaft $\left(R^{2}\right.$-Änderung von .098; $\left.p<.01\right)$. Allerdings zeigen sich keine signifikanten Wirkungen einer einzelnen Variable, sondern nur von der Tendenz her mit den Annahmen übereinstimmende Effekte - in erster Linie durch den wahrgenommenen Einfluss auf männliche Freunde/Bekannte. Bei Einschätzungen zur Empfänglichkeit von Männern im Allgemeinen spricht der Wert sogar gegen die Annahmen. Ähnliche Ergebnisse sind bei der Bereitschaft zu Schönheitsoperationen beobachtbar: Der Wert für den wahrgenommen Einfluss auf Männer im Allgemeinen ist dort signifikant - allerdings entgegengesetzt zur Hypothese: Je höher die eingeschätzte Wirkung der Bilder auf Männer im Allgemeinen, desto geringer die Bereitschaft der Frauen zu einer Schönheitsoperation $(\beta=-.32 ; p<.05)$. 
Tabelle 2: Hierarchische Regressionen - Einflïsse auf Absichten zur Änderung des eigenen Aussehens

\begin{tabular}{|c|c|c|c|}
\hline & $\begin{array}{c}\text { Franen: } \\
\text { Franenbilder } \\
\text { AV: Diät } \\
(n=135) \\
\beta\end{array}$ & $\begin{array}{c}\text { Franen: } \\
\text { Franenbilder } \\
\text { AV: Schönheits- } \\
\text { OP } \\
(n=135) \\
\beta\end{array}$ & $\begin{array}{c}\text { Männer: } \\
\text { Männerbilder } \\
\text { AV: Sport } \\
(n=54) \\
\beta\end{array}$ \\
\hline \multicolumn{4}{|l|}{ Block 1} \\
\hline $\begin{array}{l}\text { Zufriedenheit mit dem eigenen } \\
\text { Aussehen }\end{array}$ & $-.35 \% * \%$ & $-.31 * * *$ & $.53 * * 6$ \\
\hline Sensibilität bzgl. des eigenen Aussehens & .11 & .09 & .07 \\
\hline Bewertung des Aussehens der Models & $.19 *$ & .02 & .09 \\
\hline $\begin{array}{l}\text { Interesse an Prominenten/Klatsch \& } \\
\text { Tratsch }\end{array}$ & $\begin{array}{l}.02 \\
216 * * * \%\end{array}$ & $\begin{array}{l}.25 * * \\
212 * *\end{array}$ & $\begin{array}{l}.07 \\
269 * *\end{array}$ \\
\hline 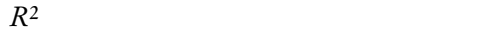 & $216 \% * \%$ & $.212^{* *}$ & $.269^{* *}$ \\
\hline \multicolumn{4}{|l|}{ Block 2} \\
\hline $\begin{array}{l}\text { Wahrg. Einfluss auf die eigene Person } \\
\text { Wahrg. Einfluss auf weibl. Freunde/ }\end{array}$ & .14 & .17 & -.15 \\
\hline $\begin{array}{l}\text { Bekannte } \\
\text { Wahrg. Einfluss auf männl. Freunde/ }\end{array}$ & .16 & .05 & $.52 *$ \\
\hline Bekannte & 24 & .15 & -.11 \\
\hline $\begin{array}{l}\text { Wahrg. Einfluss auf Frauen im } \\
\text { Allgemeinen }\end{array}$ & .01 & .07 & -.16 \\
\hline $\begin{array}{l}\text { Wahrg. Einfluss auf Männer im } \\
\text { Allgemeinen } \\
R^{2} \text { Änderung }\end{array}$ & $\begin{array}{l}-.21 \\
.098 * *\end{array}$ & $\begin{array}{l}-.32 * \\
.065\end{array}$ & $\begin{array}{l}.37 \\
.212 * *\end{array}$ \\
\hline$R^{2}$ gesamt & $.314 \% * \%$ & $.277 \% * \%$ & $.481 * * *$ \\
\hline
\end{tabular}

Alle Items waren positiv formuliert (Lesebeispiel: Ein positives Vorzeichen bedeutet: Je größer der wahrgenommene Einfluss auf die eigene Person, desto eher die Bereitschaft zu einer Schönheitsoperation); $* \mathrm{p}<.05 ; * \mathrm{p}<.01 ; * * \mathrm{p}<.001 ; \mathrm{AV}=$ Abhängige Variable

Bei den befragten Männern ist ein hypothesenrelevanter Wert signifikant: Ihre Motivation, Sport zu treiben, um den Körper zu trainieren, hängt von dem Einfluss ab, den sie den idealisierten Männerbildern auf ihre weiblichen Freunde und Bekannten unterstellen $(\beta=.52 ; p<.05)$. Recht hoch, aber nicht signifikant ist die Bedeutung des wahrgenommenen Einflusses der Bilder auf Männer im Allgemeinen. ${ }^{6}$

Hypothese 4a zur Bedeutung des Einflusses auf die eigene Person kann daher nicht bestätigt werden. Nur bei den befragten Frauen zeigen sich (in zwei von drei Messvarianten der abhängigen Variable) Resultate, die von der Tendenz her für die Hypothese sprechen. Hypothese $4 \mathrm{~b}$ zur Bedeutung des Einflusses auf andere Personen lässt sich für

6 Alternativ wurden hierarchische Regressionen gerechnet, in denen jeweils entweder der wahrgenommene Einfluss auf die eigene Person in einem zweiten Block und die wahrgenommenen Einflüsse auf andere Personengruppen als dritter Block berücksichtigt wurden oder die beschriebene Reihenfolge umgekehrt wurde. Die Ergebnisse unterschieden sich nur wenig von denen der ausführlich dargestellten Regressionen. 
die befragten Frauen nicht bestätigen, für die Männer nur bei einer Variante der abhängigen Variable - und dort mit Blick auf Frauen in der unmittelbaren Umgebung.

\section{Diskussion}

In der Studie wurden Wirkungen der Darstellung attraktiver Medienakteure untersucht. Als theoretischer Ansatz diente der Third-Person-Effekt. Überprüft wurde, ob sich Differenzen in der Wahrnehmung des Einflusses auf die eigene Person und auf andere Personengruppen ergeben. Zudem wurde ermittelt, ob Einflusswahrnehmungen Folgen für die Bereitschaft haben, das eigene Aussehen zu ändern. Anders als in bisherigen Studien wurden neben Frauen auch Männer befragt. Eingeschätzt werden sollten die Einflüsse auf andere Frauen und auf andere Männer - Basis waren entweder Bilder weiblicher oder männlicher Models.

Folgende Kernergebnisse sind hervorzuheben: 1. Dritte Personen des eigenen Geschlechts werden als anfälliger für Wirkungen von Bildern mit Models des eigenen Geschlechts eingeschätzt als die eigene Person. Dies bestätigt die generelle Annahme zur Third-Person-Perception. Die sogenannten zweiten Personen, also Freund/inn/e/n und Bekannte, werden allerdings im Vergleich zur eigenen Person nicht als spürbar beeinflussbarer gehalten. 2. Die Befragten geben an, von den Models des eigenen Geschlechts stärker beeinflusst zu werden als von Models des anderen Geschlechts. 3. Frauen schätzen den Einfluss von Bildern weiblicher Models auf andere Frauen im Vergleich zum Einfluss der Bilder auf andere Männer als sehr viel höher ein. Männer gehen dagegen mit Blick auf Männerbilder nicht von großen Unterschieden in der Wirkung auf andere Frauen und andere Männer aus. 4. Einflusswahrnehmungen haben nur sehr vereinzelt Folgen für Absichten, das eigene Äußere zu ändern - und dann auch nicht immer in der postulierten Richtung. Neben Effekten, die von der Tendenz her mit den Annahmen übereinstimmen, zeigt sich nur eine hypothesenkonforme und zugleich signifikante Wirkung einer Einzelvariable.

Zur besseren Einordnung der Ergebnisse muss auf einige Limitationen der Studie hingewiesen werden: Zum einen wurden den Befragten entweder Frauen- oder Männerbilder präsentiert und dazu Fragen gestellt. Dies schränkt die Vergleichbarkeit aller Ergebnisse etwas ein. In Studien mit einem Within-Subject-Design könnte dies zukünftig umgangen werden. Dort wären indes methodische Probleme wie etwa die Eintönigkeit des Befragungsvorgangs angesichts einer Vielzahl von Third-Person-Items zu klären. Zum anderen ist zu bedenken, dass die abgebildeten Frauen insgesamt für attraktiver gehalten wurden als die abgebildeten Männer. Ob dies zu Verzerrungen im Antwortverhalten geführt hat, ist offen. Für die meisten der hypothesenrelevanten Ergebnisse ist dieses Problem jedoch eher nachrangig, weil weniger der Vergleich von Frauen- und Männerbildern im Vordergrund stand, sondern zum Beispiel der Vergleich unterschiedlicher Personengruppen, die identische Bilder gesehen hatten.

Die Untersuchung war experimentell angelegt. Den Befragten wurden jeweils vier Bilder von Fotomodellen als Stimuli präsentiert. Obwohl diese Bilder beispielsweise aus realen Anzeigen entnommen wurden, ist die Übertragbarkeit der Resultate auf alltägliche Rezeptionsprozesse nicht ohne Weiteres möglich. Die dargestellten Models können außerdem nicht mit anderen medial auftretenden Personen, wie etwa Seriendarstellern, gleichgesetzt werden. Auch dies schränkt die Aussagekraft der Befunde ein. Ergänzungen durch weitere quantitative wie qualitative Studien wären daher sinnvoll. Dort könnte auch untersucht werden, ob in dieser Studie nicht beachtete Faktoren - wie Selektionsprozesse, Nutzungsmotive oder geschlechterspezifische Einstellungen zur medialen 
Präsentation von Frauen und Männern - bedeutsam für indirekte Wirkungen einer Rezeption idealisierter Mediencharaktere sind.

Zur Diskussion der Ergebnisse: Die im Zuge der Berechnungen zur ersten Hypothese ermittelten Wahrnehmungsdifferenzen zwischen erster Person und dritten Personen sind nicht überraschend. Das Wissen um die Differenzen ist aber hilfreich, etwa für eine präventive Kommunikation zur Verhinderung von Unzufriedenheit mit dem eigenen Aussehen oder gar Essstörungen. Neben der Tatsache, dass die Medienakteure nicht dem durchschnittlichen Aussehen in der Bevölkerung entsprechen, kann so ein weiterer Aspekt vermittelt werden: Ein möglicherweise vorhandener Wunsch nach einer Veränderung des eigenen Aussehens basiert im Einzelfall auch auf Vorstellungen darüber, dass andere Menschen durch idealisierte Medienbilder beeinflusst werden. Diese unterstellten Einflüsse sind jedoch offensichtlich übertrieben. So überschätzen Frauen vermutlich die Wirkungen von Frauenbildern auf die Zufriedenheit anderer Frauen mit deren Aussehen; und Männer überschätzen den Einfluss der Männerbilder auf andere Männer. Wenn es gelingt, zum Beispiel jungen Frauen zu verdeutlichen, dass sich andere Frauen in ihrer Körperzufriedenheit etwa von medialen Schlankheitsidealen weniger beeinflussen lassen als vermutet, kann dies unter Umständen den Druck mindern, den Frauen mit Blick auf die eigene Person verspüren.

Der Einbezug von Männern in die Studie (als Befragte, als dargestellte Models und als Personengruppen, denen Einflüsse zugeschrieben werden mussten) hat zu Ergebnissen geführt, die die bereits vorhandenen Erkenntnisse zu den vermuteten Einflüssen unter Frauen ergänzen und an einigen Stellen kontrastieren. Auch Männer sehen sich selbst als durchaus empfänglich für Wirkungen einer medialen Präsentation männlicher Models. Und Männern wird diese Empfänglichkeit zudem von anderen Personen unterstellt. Allerdings sind die selbst angegebenen sowie die von anderen unterstellten Einflüsse auf die Körperzufriedenheit bei den Frauen insgesamt größer.

In Bilanzierungen anderer Studien wurde vermutet, dass Frauen gerade anderen Männern eine größere Beeinflussung - auch und vor allem durch Frauenbilder - zuweisen könnten als anderen Frauen, weil sie Männern beispielsweise unterstellen, die idealisierten Medienakteure als Abbild der Realität zu sehen (z. B. Chia 2009). Diese Vermutung korrespondiert zudem mit Annahmen zur Rolle der sozialen Distanz bei indirekten Medienwirkungen, wonach immer die am weitesten entfernte Gruppe für am stärksten beeinflussbar gehalten wird (z. B. Meirick 2005). Diese Ergebnisse zeigen sich in der vorliegenden Studie nicht. Frauen werden von den befragten Frauen (aber auch von den befragten Männern) für wesentlich anfälliger durch die Frauenbilder gehalten als Männer. Dahingegen unterscheidet sich der vermutete Einfluss der Männerbilder auf andere Frauen und Männer nach Ansicht der männlichen (aber auch der weiblichen) Befragten nicht so sehr. Dieses Ergebnis hängt auch stark mit dem zusammen, was in der Studie gemessen wurde. Anders als etwa bei Chia (2009) wurde nicht abgefragt, welche Einflüsse anderen Personen auf deren allgemeine Schönheitsideale unterstellt werden. Vielmehr wurden Auswirkungen auf die Zufriedenheit mit dem Aussehen fokussiert. Dass anderen Frauen unterstellt wird, sie würden darin durch Bilder weiblicher Models eher beeinflusst als durch Bilder männlicher Models, ist daher wenig erstaunlich. Dass umgekehrt die Differenz zwischen zugeschriebenen Einflüssen auf andere Frauen und andere Männer nicht allzu groß ist, wenn Männerbilder präsentiert wurden, ist hingegen schon eher beachtlich.

$\mathrm{Zu}$ erwägen ist, ob hier stereotype Vorstellungen über eine insgesamt größere Beeinflussbarkeit von Frauen bezüglich ihres Aussehens eine wichtige Rolle spielen. Ihnen könnte etwa unterstellt worden sein, Äußerlichkeiten eine größere Bedeutung beizu- 
messen als Männer. Sie könnten auch als stärkere Nutzer bestimmter Medienangebote - wie etwa Modezeitschriften - gesehen werden, woraus automatisch eine größere Anfälligkeit für Wirkungen abgeleitet wird. In weiteren Untersuchungen wird zu überprüfen sein, ob diese Spekulationen haltbar sind. Solche Vermutungen könnten aber auch ein Grund dafür sein, dass sich gerade die öffentliche Diskussion über die Effekte idealisierter Medienbilder vor allem auf die Frage konzentriert, welche negativen Wirkungen auf weibliche Rezipienten zu befürchten sind. Dies kann dazu führen, Wirkungen auf männliche Rezipienten außer Acht zu lassen. Einen Grund, sie jedoch ebenfalls zu beachten, liefert die vorliegende Studie: Die Antworten der männlichen Befragten deuten wie erwähnt darauf hin, dass sie sich in ihrer eigenen Körperzufriedenheit durchaus von den Männerbildern beeinflusst fühlen.

Ein weiterer Punkt betrifft die zweiten Personen, also nicht die anonyme Masse der dritten Personen, sondern die Freund/inn/e/n und Bekannten der Befragten. Sie werden in vielen Studien, gerade zum Influence-of-Presumed-Media-Influence, nicht bedacht. Diese Untersuchung zeigt, dass es sich lohnt, sie einzubeziehen. So wird den gleichgeschlechtlichen Freund/inn/en und Bekannten oft gar kein größerer Einfluss zugeschrieben als sich selbst. Auch dieses Resultat durchbricht die Vermutung, dass mit größerer Entfernung von der eigenen Person stets eine größere Wirkungsanfälligkeit unterstellt wird.

Zudem sind die zweiten Personen in einem Fall bedeutsam für die Folgen unterstellter Wirkungen einer Rezeption attraktiver Medienakteure: Die Absicht von Männern, Sport zu treiben, um den eigenen Körper zu trainieren, wird signifikant bestärkt, wenn den Bildern männlicher Models eine hohe Wirkung auf weibliche Freunde und Bekannte zugesprochen wird. In der Tendenz zeigen sich auch Konsequenzen bei den befragten Frauen, etwa wenn sie angeben sollten, ob sie Ernährungsformen wie eine Diät einhalten: Hier war dann der wahrgenommene Einfluss der Frauenbilder auf männliche Freunde und Bekannte relevant. Das eigene Aussehen ist ein privates Thema, bei dem folglich Überlegungen mit Blick auf Freunde und Bekannte belangvoll sein können.

Die Wahrnehmung von Wirkungen auf die Allgemeinheit scheint dagegen weniger wichtig zu sein, um daraus Konsequenzen der in der Befragung thematisierten Art zu ziehen. Auffällig ist zudem, dass der wahrgenommene Einfluss auf die eigene Person höchstens tendenziell weitergehende Folgen hatte. Die Resultate stimmen nicht mit Teilergebnissen anderer Studien (z. B. Park 2005) überein. Dies könnte ebenfalls daran liegen, dass nicht vermutete Wirkungen auf Schönheitsideale als unabhängige Variable dienten, sondern vermutete Wirkungen auf die Zufriedenheit mit dem Aussehen. Es ist offensichtlich keine Selbstverständlichkeit, Vermutungen zur Unzufriedenheit anderer Personen mit eigenen Konsequenzen in Beziehung zu setzen. Insofern war diese Messvariante für eine Erfassung der Wahrnehmungsdifferenzen wohl eher geeignet als zur Überprüfung von Folgen der Wahrnehmungen. Angesichts dieser Messung ist es umso bemerkenswerter, dass vermutete Wirkungen auf Freunde bzw. Bekannte des jeweils anderen Geschlechts zumindest teilweise relevante Auswirkungen hatten. In künftigen Studien sollte jedoch zusätzlich die bloße Wahrnehmung von Wirkungen auf Schönheitsideale berücksichtigt werden - inklusive einer Messung möglicher Folgen dieser Wahrnehmung auf der Individualebene. Darüber hinaus könnten vermutete Wirkungen einer Darstellung attraktiver Medienakteure bezüglich noch negativerer Einflüsse erfasst werden - zum Beispiel hinsichtlich der Gefahren, die der eigenen Person und anderen Personengruppen für die Entstehung von Essstörungen zugeschrieben werden. 


\section{Literatur}

Bandura, A. (2002). Social Cognitive Theory of Mass Communication. In J. Bryant \& D. Zillmann (Hrsg.), Media Effects: Advances in Theory and Practice (S. 121-154). Mahwah, NJ: Erlbaum.

Baumann, E. (2009). Die Symptomatik des Medienhandelns. Zur Rolle der Medien im Kontext der Entstehung, des Verlaufs und der Bewältigung eines gestörten Essverhaltens. Köln: Halem.

Bissell, K. L. \& Zhou, P. (2004). Must-see TV or ESPN: Entertainment and Sports Media Exposure and Body-Image Distortion in College Women. Journal of Communication, 54, 5-21.

Botta, R. A. (1999). Television Images and Adolescent Girls' Body Image Disturbance. Journal of Communication, 49, 22-41.

Chia, S. C. (2007). Third-Person Perceptions about Idealized Body Image and Weight-loss Behavior. Journalism \& Mass Communication Quarterly, 84, 677-694.

Chia, S. C. (2009). When the East Meets the West: An Examination of Third-Person Perceptions about Idealized Body Image in Singapore. Mass Communication and Society, 12, 423-445.

Chia, S. C., Lu, K. H. \& McLeod, D. M. (2004). Sex, Lies, and Video Compact Disc. A Case Study on Third-Person Perception and Motivations for Media Censorship. Communication Research, 31, 109-130.

Choi, Y. Y., Leshner, G. \& Choi, J. (2008). Third-Person Effects of Idealized Body Image in Magazine Advertisements. American Behavioral Scientist, 52, 147-164.

Daszkowski, A. (2003). Das Körperbild bei Frauen und Männern. Evolutionstheoretische und kulturelle Faktoren. Marburg: Tectum.

David, P. \& Johnson, M. A. (1998). The Role of Self in Third-Person Effects about Body Image. Journal of Communication, 48, 37-58.

David, P., Morrison, G., Johnson, M. A. \& Ross, F. (2002). Body Image, Race, and Fashion Models. Social Distance and Social Identification in Third-Person Effects. Communication Research, 29, 270-294.

Davison, W. P. (1983). The Third-Person Effect in Communication. Public Opinion Quarterly, 47, 1-15.

Festinger, L. (1954). A Theory of Social Comparison Processes. Human Relations, 7, 117-140.

Fouts, G. \& Burggraf, K. (1999). Television Situation Comedies: Female Body Images and Verbal Reinforcements. Sex Roles, 40, 473-481.

Gerbner, G., Gross, L., Morgan, M., Signorelli, N. \& Shanahan, J. (2002). Growing up with Television: The Cultivation Process. In J. Bryant \& D. Zillmann (Eds.), Media effects: Advances in Theory and Practice (S. 43-68). Mahwah, NJ: Erlbaum.

Grabe, S., Ward, L. M. \& Hyde, J. S. (2008). The Role of the Media in Body Image Concerns among Women: A Meta-Analysis of Experimental and Correlational Studies. Psychological Bulletin, 134, 460-476.

Greenberg, B. S., Eastin, M., Hofshire, L., Lachlan, K. \& Brownell, K. D. (2003). Portrayals of Overweight and Obese Individuals on Commercial Television. American Journal of Public Health, 93, 1342-1348.

Gunther, A. C., Bolt, D., Borzekowski, D. L. G., Liebhart, J. L. \& Dillard, J. P. (2006). Presumed Influence on Peer Norms: How Mass Media Indirectly Affect Adolescent Smoking. Journal of Communication, 56, 52-68.

Gunther, A. C., Perloff, R. M. \& Tsfati, Y. (2007). Public Opinion and the Third-Person Effect. In W. Donsbach \& M. W. Traugott (Eds.), The SAGE Handbook of Public Opinion Research (S. 184-191). Thousand Oaks, CA: Sage.

Gunther, A. C. \& Storey, J. D. (2003). The Influence of Presumed Influence. Journal of Communication, 53, 199-215.

Harrison, K. (2001). Ourselves, our Bodies: Thin-ideal Media, Self-Discrepancies, and Eating Disorder Symptomatology in Adolescents. Journal of Social and Clinical Psychology, 20, 289-323.

Harrison, K. (2009). Media and the Body. In R. L. Nabi \& M. B. Oliver (Eds.), The SAGE Handbook of Media Processes and Effects (S. 393-407). Thousand Oaks, CA: Sage.

Harrison, K. \& Cantor, J. (1997). The Relationship between Media Consumption and Eating Disorders. Journal of Communication, 47, 40-66. 
Huck, I. \& Brosius, H.-B. (2007). Der Third-Person-Effekt - Über den vermuteten Einfluss der Massenmedien. Publizistik, 52, 355-374.

Jones, D. C. (2001). Social Comparison and Body Image: Attractiveness Comparisons to Models and Peers among Adolescent Girls and Boys. Sex Roles, 45, 645-664.

Law, C. \& Labre, M. P. (2002). Cultural Standards of Attractiveness: A Thirty-year Look at Changes in Male Images in Magazines. Journalism \& Mass Communication Quarterly, 79, 697-711.

McCroskey, L. L., McCroskey, J. C. \& Richmond, V. P. (2006). Analysis and Improvement of the Measurement of Interpersonal Attraction and Homophily. Communication Quarterly, 54, 1-31.

Meirick, P. C. (2005). Rethinking the Target Corollary: The Effects of Social Distance, Perceived Exposure, and Perceived Predispositions on First-Person and Third-Person Perceptions. Communication Research, 32, 822-843.

Milkie, M. A. (1999). Social Comparisons, Reflected Appraisals, and Mass Media: The Impact of Pervasive Beauty Images on Black and White Girls' Self Concepts. Social Psychology Quarterly, 62, 190-210.

Park, S. Y. (2005). The Influence of Presumed Media Influence on Woman's Desire to be Thin. Communication Research, 32, 594-614.

Pope, H. G., Gruber, A. J., Mangweth, B., Bureau, B., deCol, C., Jouvent, R. \& Hudson, J. I. (2000). Body Image Perception among Men in Three Countries. American Journal of Psychiatry, 157, 1297-1301.

Rossmann, C. \& Brosius, H.-B. (2005). Vom hässlichen Entlein zum schönen Schwan? Zur Darstellung und Wirkung von Schönheitsoperationen im Fernsehen. Medien E Kommunikationswissenschaft, 53, 507-532.

Spitzer, B. L., Henderson, K. A. \& Zivian, M. T. (1999). Gender Differences in Population versus Media Body Sizes: A Comparison over Four Decades. Sex Roles, 40, 545-565.

Strauß, B. \& Appelt, H. (1983). Ein Fragebogen zur Beurteilung des eigenen Körpers. Diagnostica, $29,145-164$.

Sun, Y., Pan, Z. D. \& Shen, L. J. (2008). Understanding the Third-Person Perception: Evidence from a Meta-Analysis. Journal of Communication, 58, 280-300.

Taddicken, M. (2008). Methodeneffekte bei Web-Befragungen. Einschränkungen der Datengüte durch ein "reduziertes Kommunikationsmedium"? Köln: Halem.

Tal-Or, N., Tsfati, Y. \& Gunther, A. C. (2009). The Influence of Presumed Media Influence. Origins and Implications of the Third-Person Perception. In R. L. Nabi \& M. B. Oliver (Eds.), The SAGE Handbook of Media Processes and Effects (S. 99-112). Thousand Oaks, CA: Sage.

Thomsen, S. (2002). Health and Beauty Magazine Reading and Body Shape Concerns among a Group of College Woman. Journalism E Mass Communication Quarterly, 79, 988-1007.

Xu, J. \& Gonzenbach, W. J. (2008). Does a Perceptual Discrepancy Lead to Action? A Meta-Analysis of the Behavioural Component of the Third-Person Effect. International Journal of Public Opinion Research, 20, 375-385.

\section{Anhang}

In der Studie verwendetes Bildmaterial. Den Befragten wurden entweder die vier Frauenbilder oder die vier Männerbilder präsentiert. Jedes Bild war auf einer Seite des OnlineFragebogens zu sehen. Die Bilder wurden in der hier dargestellten Reihenfolge präsentiert.
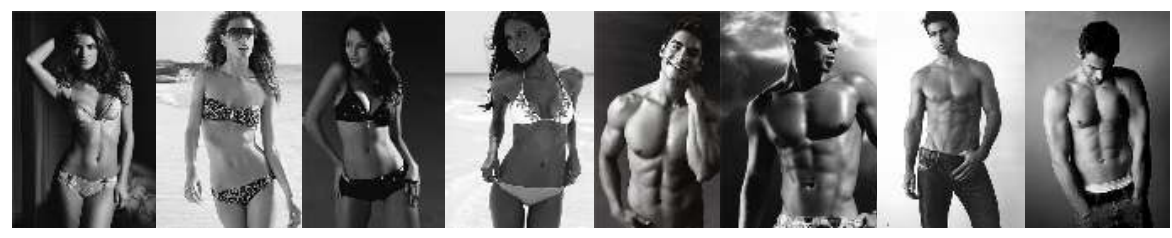